\title{
Fulminant Neuroleptic Malignant Syndrome Induced by Low Doses of Quetiapine in an Old Patient
}

\author{
Tuba Özcan ${ }^{1}$, Nilay Taş ${ }^{1}$, Esra Yancar Demir ${ }^{1}$, Murat Doğan İşcanl1 ${ }^{1}$, Özlem Özdemir ${ }^{1}$, Osman \\ Bektaş², Arzu Altunçekiç Yıldırım ${ }^{1}$ \\ ${ }^{1}$ Ordu University, School of Medicine, Ordu/ Turkey \\ ${ }^{2}$ Ordu University, Training and Research Hospital, Ordu/ Turkey
}

Received: 06 November 2015 accepted: 27 March 2016/ published online: 27 April 2016

(C) Ordu University Institute of Health Sciences, Turkey, 2016

\begin{abstract}
Neuroleptic malignant syndrome (NMS), a rare but life-threatening idiosyncratic adverse reaction to antipsychotic medication. NMS is commonly seen with typical antipsychotics that also reported with atypical antipsychotic drugs.

A 81-year-old man was presented to emergency department of our hospital due to altered mental state. He had been taking quetiapine for 3 days for management of aggressive behavior. Just after treatment with 50 $\mathrm{mg} /$ day quetiapine at the previous hospital, he developed hyperthermia, altered mental status and akineticrigid Parkinsonism, and he had been transferred to our hospital. He was diagnosed with possible NMS based on history, altered mental state, rigidity, leukocytosis, hyperthermia, and increased blood level of CK. So, he was admitted to the intensive care unit immediately.

This paper reports a rare fulminant NMS induced by low doses of quetiapine. The clinical manifestations and laboratory test results corresponded to the diagnostic criteria of NMS. It must be kept in mind that even low doses of atypic antipsychotic drugs such as quetiapine can trigger NMS.

Key words: neuroleptic malignant syndrome, atypical antipsychotic, quetiapine
\end{abstract}

Address for correspondence/reprints:

Özlem Özdemir

E-mail address: ozlemtfl@hotmail.com

Phone: +090 5356819716

DOI: $10.19127 /$ mbsjohs.93967

\section{Introduction}

Neuroleptic malignant syndrome (NMS), an idiosyncratic reaction to antipsychotic medication, is characterized by hyperthermia, akinetic-rigid syndrome, altered mental state, and autonomic instability including fluctuation of blood pressure, cardiac arrhythmias, dyspnea, diaphoresis, and incontinence for review (Chopra et all, 1999; Strawn et all, 2007; Trollor et all; 2009).

Mechanisms of NMS are not fully understood but drug-induced blockade of central dopamine receptors and central dopaminergic hyponeurotransmission are thought to be pivotal in its etiology (Mann et al., 1991; Adnet et al., 2000; Strawn et al.; 2007). The syndrome has a mortality 
rate as high as $20 \%$ ( Caroff et al., 1991; Caroff et al., 1993).

However, NMS is commonly seen with typical antipsychotics that also reported with atypical antipsychotic drugs (Ananth et al., 2004; Nielsen et al., 2012). NMS has been known to be mild when it is caused by treatment with atypical antipsychotics (Kobayashi et al., 2006; Trollor et al., 2009; Woods et al., 2013; Detweiler et al., 2013).

We herein report a rare case of death caused by fulminant NMS who developed NMS after the initiation of quetiapine.

\section{Case}

A 81-year-old man was presented to emergency department of our hospital due to altered mental state. He had been taking quetiapine for 3 days for management of aggressive behavior. He had been admitted to another hospital 3 days earlier due to hallucinations and frequent loss of consciousness for brief periods of time. Just after treatment with $50 \mathrm{mg} /$ day quetiapine at the previous hospital, he developed hyperthermia, altered mental status and akinetic-rigid Parkinsonism, and he had been transferred to our hospital. On initial neurological examination in our hospital, his mental status was coma, with rigidity especially in upper extremity. There was no focal neurological deficits. His blood pressure was fluctuant and pulse elevated with $180 /$ minute heart rate, body temperature was $37.5^{\circ} \mathrm{C}$, serum creatine kinase $(\mathrm{CK})$ concentration was 4267 IU/L. WBC was 16300/dL. Routine blood chemistry showed a glucose level of $319 \mathrm{mg} /$ $\mathrm{dL}$, AST and ALT concentrations of 168 and 79 IU/L, respectively, with electrolyte levels within the normal range $(\mathrm{Na} 139 \mathrm{mEq} / \mathrm{L}, \mathrm{K} 3.7 \mathrm{mEq} / \mathrm{L}$ and $\mathrm{Cl} 98 \mathrm{mEq} / \mathrm{L}$ ). Chest X-rays showed normal findings and there was no evidence of any infective diseases in urine analysis or blood culture. Brain MRI showed age-compatible brain atrophy. $\mathrm{He}$ was diagnosed with possible NMS based on history, altered mental state, rigidity, leukocytosis, hyperthermia, and increased blood level of CK. So, he was admitted to the intensive care unit immediately. The antipsychotic drug was discontinued and he was started on treatment with bromocriptine $(7.5 \mathrm{mg}$ / day). Ice packs were used to decrease the fever. Suppotive therapy was provided to maintain hydration, stabilize blood pressure, control cardiac state, and prevent infection and secondary organ failures. He was followed together with cardiology, anesthesiology and reanimation, infectious diseases, internal medicine, and psychiatry specialists. $\mathrm{He}$ was undergone nasal feeding, liquid diet, oxygen therapy and electrocardiographic monitoring. His body temperature decreased and but his mental state was still coma. CK level decreased to 1574 IU/L and WBC decreased to $10500 / \mathrm{dL}$ on the third day. Unfortunately, even aggressive resuscitation he died at the end of third day of hospitalization.

The patient described here presented with akinetic-rigid syndrome, altered mentality, hyperthermia and tachycardia. Laboratory findings, including increased serum CK concentrations, along with his clinical presentation, were consistent with NMS. The NMS occurred 3 days after starting treatment with quetiapine.

\section{Discussion}

NMS was first reported in 1960 by Delay et al (1960). The reported incidence of NMS is 0.1 to $1 \%$ and the mortality is $20 \%$ (Caroff et al., 1991; Caroff et all, 1993). In a recent report, Zou et al. reported a death due to low doses of haloperidol induced NMS in a young male diagnosed with schizophrenia (Zou et all, 2014). The onset of NMS symptoms is usually acute, and the symptoms are complex and atypical, so it is difficult to diagnose.

The most commonly used diagnostic criteria for NMS are provided in DSM-IV-TR (American Psychiatric Association. Diagnostic, 1994):

a) Severe muscle rigidity and elevated body temperature after the use of antipsychotic medications,

b) Two or more associated symptoms including diaphoresis, dysphagia, tremor, incontinence, altered mental status, mutism, tachycardia, elevated or label blood pressure, leukocytosis, and laboratory evidence of muscle damage (such as elevated CPK).

c) The symptoms cannot be explained by other drugs, neurological diseases, physical illnesses, or another mental disorder.

The exact pathogenesis of NMS is not clear. Antipsychotic drugs work by blockage of dopamine receptors. Blockade of dopamine receptors of the hypothalamus may cause autonomic dysfunction. Interaction with nigrostriatal system may result in extrapyramidal symptoms. Mesocortical dopaminergic system are result in mental symptoms of NMS. Also, antipsychotic drugs have a direct changes in muscle mitochondrial function (Mann et al., 1991; 
Adnet et al., 2000; Strawn et al., 2007). In our patient, he presented to our emergency department with coma.

In the acute phase, patients with NMS experience fever and dehydration, so the therapy must include rehydration and correction of electrolyte imbalances. The antipyretic drugs are usually ineffective cause of the dysfunction of the thermoregulatory center, so fever needs to be lowered with ice packs (Delay et al., 1960). Our patient had subfebrile fever, however, in case of considering as NMS, we can say that atypical antipsychotics may lead to atypical NMS.

All of the dopamine antagonists may be associated with NMS, whereas atypical antipsychotics have a lower risk of inducing NMS than typical ones, because of looser binding affinity to D2 receptors in the basal ganglia and hypothalamus (McEwan et al., 2000; Ananth et al., 2004). In this case, the patient developed possible NMS after taking quetiapine, an atypical antipsychotic drug which is a dibenzothiazepine derivative. Firstly, Kobayashi et al. reported a quetiapine induced NMS case in DLB ( Kobayashi et al., 2006) In the literature, there are a few case reports suggesting that quetiapine can cause symptoms such as those observed in the present case (Ananth et al., 2004; Kobayashi et al.,2006; Detweiler et al., 2013; Woods et al., 2013).

Risk factors of NMS include rapid changes in drug dosages, hyponatremia, pre-existing medical and neurological disorders, iron deficiency, and exhaustion or dehydration. In all ages of both genders are susceptible to NMS (Trollor et al., 2009). The symptoms of NMS usually begin within 2 weeks of the initiation or change in dose of drug treatment. Approximately $16 \%$ of cases occur within $24 \mathrm{~h}$ following the initiation of drug treatment (Ananth et al., 2004; Strawn et al., 2007; Trollor et al., 2009). In our case, the patient had an episode and a rapid death thereafter, which can be considered as a fulminant type of NMS. NMS is associated with many antipsychotic drugs, and quetiapine is one of them. In this present case, the patient received $25 \mathrm{mg}$ of quetiapine which is a relatively low dose when compared with doses in other NMS cases induced by quetiapine in the literature (Kobayashi et al., 2006; Detweiler et al., 2013; Woods et al., 2013). Anyway, NMS is an idiosyncratic disorder, and the onset is not related with the duration or amount of doses (Adnet et al., 2000).

We conclude that each antipsychotic drug can lead to NMS per dose. Especially in older patients we must be careful for prescribing antipsychotics. In geriatric population, whether we suspect dementia and behavioral disorders, we should consider the sensitivity of this age group, and primarily prefer the antidepressants and behavioral therapy. Also, delirium should be a differential diagnosis in patients with dementia and altered state. In our case, even fever, CK, WBC were decreased, the patient have died. So, we cannot know the initial diagnosis and triggers.

\section{Conclusion}

In conclusion, this paper reports a rare fulminant NMS induced by low doses of quetiapine. The clinical manifestations and laboratory test results corresponded to the diagnostic criteria of NMS. It must be kept in mind that even low doses of atypic antipsychotic drugs such as quetiapine can trigger NMS. And so, clinicians should pay attention in beginning upon atypical antipsychotic drugs in patients with dementia. This report also emphasizes the importance of NMS following antipsychotic medication as a probable cause of death. Sometimes even low doses of antipsychotic drugs can cause a fatal course.

Informed Consent: Patient release form was obtained.

Peer-review: Externally peer-reviewed.

Author Contributions: Concept T.Ö, N.T Design E.D, M.İ, Supervision Ö.Ö, Funding E.D, O.B, Materials T.Ö, N.T, Data Collection and/or Processing T.Ö, N.T, A.Y, Analysis and/ or İnterpretation Ö.Ö, Literature Review T.Ö, N.T, Writing T.Ö, Ö.Ö, Critical Review Ö.Ö, A.Y

Conflict of Interest: No conflict of interest was declared by the authors.

Financial Disclosure: The authors declared that this study hasn't received no financial support. 


\section{References}

Adnet P, Lestavel P, Krivosic-Horber R. Neuroleptic malignant syndrome. Br J Anaesth 2000; 85: 129-35.

American Psychiatric Association. Diagnostic and Statistical Manual of Mental Disorders, 4th ed. Washington, DC: American Psychiatric Association 1994.

Ananth J, Parameswaran S, Gunatilake S, Burgoyne K, Sidhom T. Neuroleptic malignant syndrome and atypical antipsychotic drugs. $\mathrm{J}$ of Clin Psychiatry 2004; 65: 464-70.

Caroff SN, Stephan CM, Lazarus A, Sullivan K, MacFadden W, Neuroleptic malignant syndrome: diagnostic issues. Psychiatric Annals 1991; 21: 130-47.

Caroff SN, Mann SC: Neuroleptic malignant syndrome. Medical Clinics of North America 1993; 77: 185-202.

Chopra MP, Prakash SS, Raguram R. The neuroleptic malignant syndrome: an Indian experience. Compr Psychiatry 1999; 40: 19-23.

Delay J, Pichot P, Lemperiere T, Elissalde B, Peigne F. A non-phenothiazine and nonreserpine major neuroleptic, haloperidol, in the treatment of psychosis. Ann Med Psychol (Paris). 1960; 118: 145-52.

Detweiler MB, Sullivan K, Sharma TR, Kim KY, Detweiler JG. Case Reports of Neuroleptic Malignant Syndrome in Context of Quetiapine Use. Psychiatr Q 2013; 84(4): 523-41.

Kobayashi A, Kawanishi C, Matsumura T, Kato D, Furukawa R, Kishida I, et al. Quetiapineinduced neuroleptic malignant syndrome in dementia with Lewy bodies: A case report. Prog Neuro-Psychopharmacology Biol Psychiatry 2006; 30: 1170-2.

Mann SC, Caroff SN, Lazarus A. Pathogenesis of neuroleptic malignant syndrome. Psychiatr Ann 1991; 21: 175-80.

McEwan B. Allostasis and allostatic load. Implication for neuropsychopharmacology. Neuropsychopharmacology 2000; 22: 108-24.

Nielsen RE, Jensen SOW, Nielsen J. Neuroleptic malignant syndrome-an 11-year longitudinal case-control study. Can J Psychiatry 2012; 57: 512-18.

Strawn JR, Keck Jr PE, Caroff SN. Neuroleptic malignant syndrome. Am J Psychiatry 2007; 164: 870-6.

Trollor JN, Chen X, Sachdev PS. Neuroleptic malignant syndrome associated with atypical antipsychotic drugs. CNS Drugs 2009; 23: 47792.
Woods G, et al. Neuroleptic malignant syndrome associated with quetiapine and venlafaxine use: a case report and discussion. Ther $\mathrm{Adv}$ Psychopharmacol 2013; 3(1): 53-5.

Zou D, Shao Y, Qin Z, Zhang J, Liu N, Li Z, et al. Death due to fulminant neuroleptic malignant syndrome induced by low doses of haloperidol: A rare case. Journal of Forensic and Legal Medicine 2014; 24: 12-14. 